\title{
Suicide Ideation and Drug Craving in Patients Under Methadone Maintenance Treatment (MMT)
}

\author{
Nour-Mohammad Bakhshani $\mathbb{D}^{1}{ }^{1}$, Shahab Lotfinia $\left(\mathbb{B}^{2,}{ }^{2,}\right.$, Abolfazl Payandeh ${ }^{3}$ and Zohre Soorgi ${ }^{2}$ \\ ${ }^{1}$ Children and Adolescent Health Research Center, Zahedan University of Medical Sciences, Zahedan, IR Iran \\ ${ }^{2}$ Faculty of Medicine, Zahedan University of Medical Sciences, Zahedan, IR Iran \\ ${ }^{3}$ Department of Epidemiology \& Biostatistics, Health Promotion Research Center, School of Health, Zahedan University of Medical Sciences, Zahedan, IR Iran \\ "Corresponding author: Faculty of Medicine, Zahedan University of Medical Sciences, ZAUMS Main Campus, Zahedan, IR Iran. Tel: +98-9107797226. Email: \\ sh.lotfinia@zaums.ac.ir \\ Received 2019 March 17; Revised 2019 July 02; Accepted 2019 July 31.
}

\begin{abstract}
Background: Currently, methadone maintenance treatment (MMT) is a well-known method, for treating opiate dependence in many parts of the world. Despite being in MMT programs, craving has an important role in relapses. Substance abusers are at high risk of suicide, and suicidal ideation is a key factor for predicting suicide attempts.

Objectives: This study was conducted to determine suicide ideation and drug craving and their predictors in patients under MMT. Patients and Methods: This cross-sectional study was conducted on 234 patients under MMT at Baharan Psychiatric Center of Zahedan, Iran. Participants completed a set of self-report standard questionnaires, including Beck's Depression Inventory, Beck's Anxiety Inventory, Borderline Personality Scale, Social Support Appraisals Scale, the Communication Skills Inventory, and Craving Inventory. Bivariate correlation analyses and linear regression model were employed to identify the predictors of suicide ideation and craving.

Results: Occupational status, suicide attempts, suicide in the family, symptoms of borderline personality, and communication skills were significant predictors for suicide ideation. The duration of MMT and borderline personality symptoms were also important determinants of craving.

Conclusions: Since people under MMT have high suicide ideation and it can be a risk factor for suicide attempts, it is suggested to focus on early detection of suicide ideation. Mental health staff must pay attention to the risk factors for craving because it could become the cause of drop-out during treatment.
\end{abstract}

Keywords: Suicide, Craving, Methadone, Substance Related Disorders

\section{Background}

Methadone is a syntactic long-acting full $\mu$-opioid agonist that acts by parenteral and oral routes, at first synthesized as a possible painkiller in Germany in the late 1930s and 1950s in the United States. After research during 1964, it was used as a maintenance treatment for heroin addiction, and approved by the U.S. Food and Drug Administration (FDA) in 1972 (1).

Currently, methadone maintenance treatment (MMT) is an important method for the treatment of opiate dependence in many parts of the world (2). Furthermore, MMT provides a stable background dosage of opioids that blocks craving, blocks the "high" of other opioids, provides physiologic homeostasis (1), and reduces side effects, such as deaths related to heroin use (3). Methadone is orally administered and has a 24- to 36-hour half-life (4). Previous studies have shown that treatment with methadone is safe and effective (5).

Drugs have a strong physical and psychological dependency, and the vast majority of substance abusers relapse after detoxification treatment and one of the psychological factors is craving; opiate craving may play a role in the continued use of opiates, despite being in MMT. Opiate craving might alleviate withdrawal symptoms or increase desire due to euphoric effects. Craving is a subjective phenomenon experienced by many drug users and has a strong association with relapse after treatment (6), and it can be triggered by environmental factors. Despite being in MMT programs, craving has an important role in relapses. Health staff need to notice the elements that may not be created by Methadone, like non-compliance with treatment, stress, and poor recovery environment (7).

Substance abusers are at high risk of suicide, and suicide is one of the major reasons for death among opioid 
users (8). The rate of suicide attempted is greatly higher in substance abusers (with a lifetime rate of $17 \%$ to $40 \%$ ) than the general population (with a lifetime rate of 3\% to 5\%) (9). Suicide ideation is a key factor for suicide attempts, suicide ideation defined by the presence of plans and wishes to perform suicide in individuals, who have not performed any recent obvious suicide attempts (10). In the national comorbidity survey, the transition chances from suicide ideation to suicide plan were $34 \%$ and from a plan to attempt were $72 \%(11,12)$. Since substance use increases the likelihood of suicide ideation, more studies need to focus on early detection. Important interventions must be developed to prevent completed suicide $(13,14)$. Since maintenance treatment with methadone increases quality of life, it may reduce the risk of suicide (15).

\section{Objectives}

The present study aimed at assessing suicide ideation and craving and its predictors in patients at MMT.

\section{Patients and Methods}

\subsection{Participants and Procedure}

This cross-sectional study was conducted during year 2018. Participants in this study were recruited from the methadone maintenance treatment (MMT) program in Baharan MMT Clinic of Zahedan, Iran.

A total of 234 patients participated in this study, and they completed a set of self-reported questionnaires. Informed consent was obtained from all participants, and all the patient records were kept confidential. The inclusion criteria for this study was having at least elementary literacy in order to have the capability for completing the questionnaires and patients, who consumed any substance except methadone were excluded from the study. The study procedure was approved by the ethics committee of $\mathrm{Za}$ hedan University of Medical Sciences (ZUMS), Iran.

\subsection{Measurements}

The assessment included demographic characteristics, encompassed lifetime information of the type of substance use before treatment, suicide attempts, history of suicide in the family, relapses, current methadone dosage, and duration of MMT.

The Beck Depression Inventory (BDI) is a 21-item Likert scale questionnaire, which evaluates the symptoms of depression. Scores range from 0 (lowest severity) to 63 (highest severity), a score between 0 to 21 shows low depression, a score between 21 and 31 describes moderate depression and a score higher than 31 shows severe depression. The examiner can provide the test instruction in writing or verbally to the patient (16). The validity and reliability of the Persian version are 0.73 and 0.91 (17).

The Beck Anxiety Inventory (BAI) is a 21-item Likert scale, which evaluates the symptoms of anxiety. A score between 0 and 21 shows low anxiety, from 22 to 35 indicates moderate anxiety, and higher than 36 is potentially levels of clinical anxiety (18). The validity and reliability of the Persian version are 0.72 and 0.83 (19).

The Borderline Personality Scale (STB) has 22 items and was designed to assess the borderline personality from a variety of dimensions (hopelessness factor, impulsivity factor, paranoid/dissociative symptoms). Questions given a score of one (for positive answer) or zero (for negative answer) (20). The validity and reliability of the Persian version are 0.64 and 0.61 (21).

The Social Support Appraisals Scale (SS-A) is a Likert (Score 0 to 5) instrument with 23 items based explicitly on Cobb's (1976). Except for SS-A total (sum of all 23 items), three components are typically measured: family, friends, and people or others in a general way (22). The validity and reliability of the Persian version are 0.85 and 0.90 (23).

The Communication Skills Inventory of Jerabek has 34 items and is self-report. Responses are in a five-point Likert scale $(1=$ never, $2=$ seldom, $3=$ occasional, $4=$ most times, and $5=$ always). Total scores are categorized as follow: 34 to 68 , poor; 69 to 102 , moderate; $\geq 103$, and strong communication skills (24). The validity and reliability of the Persian version are 0.71 and 0.89 (25).

Beck Scale for Suicidal Ideations (BSSI) is a 19-item inventory developed to identify the severity of suicidal ideation. Items evaluate the respondent's plans for suicide (26). The scale is based on a Likert and scored from 0 to 2 . A score from 0 to 5 shows low risk, 6 to 19 high risk, and 20 to 38 very high risk of suicide (27). The validity and reliability of the Persian version are 0.76 and 0.95 (28).

Craving scale is a 20-item self-report, which measures the thoughts and temptation of drug abusers. This scale was made by Faderdy, Barafran, and Ziaee. The questions were scored based on a Likert scale, ranging from very low (1) to very high. The validity and reliability of the Persian version are 0.76 and 0.95 . The validity and reliability of the Persian version are 0.55 and $0.94(4,29)$.

\subsection{Statistical Analysis}

After collecting the data, data was entered in the SPSS (version 22) software. First, data quality was carefully evaluated, statistical analysis included Pearson correlation coefficients between two quantitative variables, Eta between nominal and internal variables, Gamma between two ordinal variables, chi-square, and Fisher exact tests were 
used for evaluation of dependency between two categorical variables. Finally, multiple regression models were used to determine the predictors for suicide ideation and craving in MMT patients. An expert performed all the statistical analyses.

\section{Results}

From a total of 234 participants, 195 (83.3\%) were male. The mean \pm standard deviation of age of participants was $39.1 \pm 9.92$. The sociodemographic data, patterns of substance use before MMT, suicide attempts, history of a family suicide, relapses, current methadone dosage, duration of MMT, suicide ideation, and craving of participants are summarized in Table 1.

There were 51 (21.8\%) patients, who had a different level of suicide ideation. According to the bivariate correlation analysis, increasing age, having a job, higher social support, higher communication skills and being male significantly reduced suicide ideation. However, being male, increased suicide attempts, a family with suicide history, higher depression, borderline personality symptoms, and higher anxiety increased suicide ideation.

There were 93 (39.7\%) patients, who had a different level of craving. According to the bivariate correlation analysis, increasing age, having a job, higher communication skills, and longer duration of MMT skills significantly reduced craving. However, being single, having a family with suicide history, higher depression, borderline personality symptoms, and higher anxiety increased craving.

Results of correlation analysis is summarized in Table 2.

A stepwise linear regression model was conducted to identify the predictors of suicide ideation and craving. The researchers also checked multi-collinearity of the predictors. The results are presented in Table 3. Having no job, history of suicide attempts, suicide in the family, borderline personality disorder symptoms and communication skills can predict $34 \%$ of the variation in suicide ideation ( $F$ $=23.92, \mathrm{P}<0.001$ ).

The shorter duration of MMT and Borderline personality disorder can predict $20 \%$ of the variation in craving $(\mathrm{F}=$ 28.04, $\mathrm{P}<0.001$ ).

Base on this predictors, Borderline personality disorder symptoms have a higher effect on suicide ideation (standard coefficient Beta $=0.27$ ) and suicide in the family has less effect (standard coefficient Beta $=0.12$ ). From the Craving predictors, Borderline personality disorder symptoms have a higher effect (standard coefficient Beta $=0.33$ ) and duration of MMT has a lower effect (standard coefficient Beta $=-0.26$ ).

\begin{tabular}{|c|c|}
\hline Variables & Values $^{\mathrm{a}}$ \\
\hline Age & $39.1 \pm 9.92$ \\
\hline Methadone dose (cc) & $13.56 \pm 5.63$ \\
\hline Duration of MMT (months) & $24.28 \pm 31.10$ \\
\hline \multicolumn{2}{|l|}{ Gender } \\
\hline Male & $195(83.3)$ \\
\hline Female & $39(16.7)$ \\
\hline \multicolumn{2}{|l|}{ Marital status } \\
\hline Single & $62(26.5)$ \\
\hline Married & $143(61.1)$ \\
\hline Divorced or widow & $29(12.4)$ \\
\hline \multicolumn{2}{|l|}{ Occupational status } \\
\hline On job & $118(50.4)$ \\
\hline jobless & $116(49.6)$ \\
\hline \multicolumn{2}{|l|}{ Education } \\
\hline Elementary & $67(28.6)$ \\
\hline Under the diploma & $90(38.5)$ \\
\hline Diploma and above & $77(31.9)$ \\
\hline \multicolumn{2}{|l|}{ Suicide attempts } \\
\hline None & $191(81.6)$ \\
\hline 1- 2 attempt & $29(12.4)$ \\
\hline$\geq 3$ attempt & $14(6)$ \\
\hline \multicolumn{2}{|l|}{ Suicide in the family } \\
\hline Have & $16(6.8)$ \\
\hline Have not & $218(93.2)$ \\
\hline \multicolumn{2}{|l|}{ Relapse } \\
\hline None & $86(36.8)$ \\
\hline$<5$ & $113(48.3)$ \\
\hline$\geq 5$ & $35(14.9)$ \\
\hline \multicolumn{2}{|l|}{ The pattern of substance use } \\
\hline Opiate & $126(53.8)$ \\
\hline Stimulants & $35(15)$ \\
\hline Opiate and stimulants & $44(18.8)$ \\
\hline Other patterns & $29(12.4)$ \\
\hline \multicolumn{2}{|l|}{ Suicide ideation } \\
\hline Low risk & $183(78.2)$ \\
\hline High risk & $35(15)$ \\
\hline Very high risk & $16(6.8)$ \\
\hline \multicolumn{2}{|l|}{ Craving } \\
\hline No & $141(60.3)$ \\
\hline Low & $37(15.8)$ \\
\hline Average & $31(13.2)$ \\
\hline High & $25(10.7)$ \\
\hline
\end{tabular}

${ }^{\mathrm{a}}$ Values are expressed as mean $\pm \mathrm{SD}$ or No. (\%).

\section{Discussion}

This study aimed at determining the rate of suicide ideation and craving and their predictors in patients at 


\begin{tabular}{|c|c|c|c|c|}
\hline \multirow{2}{*}{ Variables } & \multicolumn{2}{|c|}{ Suicide ideation } & \multicolumn{2}{|c|}{ Craving } \\
\hline & $\mathbf{r}$ & $\mathbf{P}$ & $\mathbf{r}$ & $\mathbf{P}$ \\
\hline Age & -0.17 & $<0.001$ & -0.27 & $<0.001$ \\
\hline Gender (Male) & 0.16 & 0.05 & 0.14 & 0.19 \\
\hline Suicide attempts (yes) & 0.70 & $<0.001$ & 0.15 & 0.661 \\
\hline Marital status (single) & 0.08 & 0.18 & 0.11 & 0.002 \\
\hline family suicide (yes) & 0.19 & 0.012 & 0.23 & 0.009 \\
\hline depression & 0.49 & $<0.001$ & 0.29 & $<0.001$ \\
\hline borderline & 0.48 & $<0.001$ & 0.36 & $<0.001$ \\
\hline Social support & -0.14 & $<0.001$ & -0.11 & 0.08 \\
\hline Communication skills & -0.29 & $<0.001$ & -0.18 & $<0.001$ \\
\hline Duration of MMT & -0.03 & 0.63 & -0.29 & $<0.001$ \\
\hline
\end{tabular}

\begin{tabular}{|c|c|c|c|c|c|c|}
\hline \multirow{2}{*}{ Variables } & \multicolumn{3}{|c|}{ Suicide Ideation } & \multicolumn{3}{|c|}{ Craving } \\
\hline & $\mathbf{B}^{\mathbf{a}}$ & $\operatorname{SE}(B)^{b}$ & PValue & $\mathbf{B}^{\mathbf{a}}$ & $\operatorname{SE}(B)^{b}$ & P Value \\
\hline Constant & 6.72 & 2.85 & 0.02 & 9.71 & 2.51 & $<0.001$ \\
\hline Occupational status (on job) & -2.99 & 0.83 & $<0.001$ & - & - & - \\
\hline Suicide attempts (yes) & 4.49 & 1.18 & $<0.001$ & - & - & - \\
\hline Suicide in the family (yes) & 3.66 & 1.64 & 0.03 & - & - & - \\
\hline Borderline & 0.41 & 0.09 & $<0.001$ & 1.35 & 0.24 & $<0.001$ \\
\hline Communication skills & -0.08 & 0.03 & 0.019 & - & - & - \\
\hline \multirow[t]{2}{*}{ Duration of MMT } & - & - & - & -0.17 & 0.04 & $<0.001$ \\
\hline & \multicolumn{3}{|c|}{$\mathrm{R}^{2}=34 \% ; \mathrm{F}=23.92 ; \mathrm{P}<0.001$} & \multicolumn{3}{|c|}{$\mathrm{R}^{2}=20 \% ; \mathrm{F}=28.04 ; \mathrm{P}<0.001$} \\
\hline
\end{tabular}

${ }^{a}$ Regression coefficients.

${ }^{\mathrm{b}}$ Standard error $(\mathrm{SD} / \sqrt{n})$.

MMT. After it evaluated 234 participants under MMT, the findings showed that $39.7 \%$ of the patients had different levels of craving, and $21.8 \%$ were at high or very high risk of suicide. The results showed that depression, anxiety, borderline personality disorder symptoms, lower communication skills, lower social support, having a history in family members, suicide attempts, occupation status, being female, and lower age had a correlation with suicide. Furthermore, occupational status, suicide attempts, suicide in the family, borderline personality disorder and communication skills could be a predictor for suicide ideation. Also, younger age, depression, borderline personality disorder, anxiety, lower communication skills, shorter duration of MMT, occupational status, being single, and history of suicide in the family had a correlation with craving. The duration of MMT and borderline can be a predictor for craving.
Communication skills is one of the predictors for suicide ideation in MMT patients. Communication skills allow people to communicate productively and properly with others. However, poor communication skills is a determinant of suicide ideation and can cause loneliness and more stress in these people (30). In 2017, similar research to the current study showed poor communication skills, as a factor associated with suicidal ideation (31).

Employment was an important outcome indicator in the context of addiction treatment and rehabilitation (32). A study in 2015, similar to the current study, showed there is a significant relationship between being unemployed and suicide ideation. Unemployment is a risk factor for poor mental health status and has been found to be both a significant and unique risk factor for suicidal ideation (33). The result of Blakely's study showed that the asso- 
ciation between being unemployed and suicide ideation was attributable to confounding determinants associated with the socioeconomic state and that it is barely related to mental health. Furthermore, the absence of economic independence as a result of unemployment decreases the probability of using health services properly (34).

A borderline personality disorder is another predictor for both suicide ideation and craving in patients under MMT. According to the latest researches, almost $80 \%$ of people with BPD perform at least one suicide attempt in their life and the suicide rate amongst people with BPD is 50 times higher than the general population. There are features in BPD that lead patients to suicide, such as dysregulation of emotions, impulsivity that engages them in a moment of intense emotional pain, brain abnormalities, chronic condition, comorbidity with other mental disorders and similar to our patients, substance use that causes of greater impulsivity $(35,36)$. Also, they are at a greater risk for relapse because of the inability to cope through problem-solving when they face negative emotional states and social rejection (37). Results of another study in 2015, similar to the current study, showed a positive correlation between borderline personality traits and level of craving (38).

This study showed that the duration of MMT could be a determinant for drug craving, and patients, who have been under MMT for a longer time have less craving in comparison to patients, who have been under MMT for a shorter time. A study in 2014 identified similar results based on brain activity; this study measured craving with a visual analog scale, before and after imaging and the results showed that long duration of MMT may improve craving response by adjusting the impaired function in the bilateral dorsal striatum caused by prior drug use (39).

Having a history of suicide in the family is correlated with both craving and suicide ideation. It is wellrecognized that suicidal behavior runs in families. It is possible that the transmission of suicidal behaviors in families occurs through the mediation of impulsive aggression. Considering that around 50\% of the variance is explained by genes, the role of environmental factors is important. One of the explanations can be adverse familial environments (40).

Age was another factor associated with craving in the current study. It shows that older patients tended to have less craving than younger individuals. In line with this findings, studies showed that older people are more likely to remain in treatment (8). Mood disorders are important predictors for suicidal ideation yet do not explain the transition from suicide ideation to suicide attempt. Rather, features associated with anxiety and impaired impulse control appear important for predicting suicide at- tempts. The last study on MMT patients supports the current findings that social support for MMT directly affected suicidal ideation (41). Another study on MMT patients supported the current findings that patients are depressed and anxious, and female are more likely to have suicide ideation (41).

Studies show anhedonia, low belongingness, bullying, burdensomeness, defeat, emotion dysregulation, entrapment, and social disconnection as a predictor of suicidal ideation yet these risk factors may be risk factors for suicide ideation, not suicide attempts. Increased information about the shift from suicide ideation to suicide attempts, is essential for prediction and prevention (42). Although most individuals, who have suicide ideation do not act on their thoughts, it seems that when suicidal ideation combines with the capacity to overcome the fear of death, suicide attempts may occur (43).

Most studies reported that higher dose of methadone is a protective factor for craving. This information provides guidelines for adequate methadone dosing to prevent relapses. In contrast, another evidence shows that patients in maintenance treatment are not protected from experiencing craving. Along with the current study, sufficient methadone dosage seems to be a crucial factor for the efficiency of treatment (7).

Since many factors can be associated with suicide ideation and craving, it is suggested to explore the role of other factors in future studies. Also, the factors that can predict the transmission of suicidal ideation to suicide attempts in these patients must also be considered. Since the majority of participants in this study had a lower level of socio-economic status, this research should be repeated in patients with medium and high level socio-economic status.

\subsection{Conclusions}

People under MMT, experience more pressure from their family or society and that makes them more vulnerable. They may choose suicide or relapse to drugs because of their poor ability to find a better solution, and this shows the importance of monitoring them and identifying the risk factors for suicide and relapses.

\section{Acknowledgments}

This research was supported by Zahedan University of Medical Sciences, Zahedan, Iran. The researchers appreciate the cooperation of supporters and participants. 


\section{Footnotes}

Authors' Contribution: Study concept and design: NourMohammad Bakhshani and Shahab Lotfinia; data gathering: Shahab Lotfinia; data analysis: Abolfazle Payandeh; manuscript drafting: Shahab Lotfinia and Zohre Soorgi; critical revision of the manuscript: Nour-Mohammad Bakhshani, Shahab Lotfiniai, Abolfazle Payandeh, Zohre Soorgi.

Conflict of Interests: The authors had no conflict of interest.

Ethical Approval: The Ethical Committee of ZAUMS reviewed and approved the study design and all procedures (IR.ZAUMS.REC.1397.253).

Funding/Support: This study was supported financially by Zahedan University of Medical Sciences, Zahedan, Iran.

\section{References}

1. Ries RK, Fiellin DA, Miller SC, Saitz R. The ASAM principles of addiction medicine. Philadelphia, PA: Lippincott Williams \& Wilkins; 2014.

2. Isbell $\mathrm{H}$, Vogel $\mathrm{VH}$. The addiction liability of methadon (amidone, dolophine, 10820) and its use in the treatment of the morphine abstinence syndrome. Am J Psychiatry.1949;105(12):909-14. doi: 10.1176/ajp.105.12.909. [PubMed:18127077].

3. Gunne LM, Gronbladh L. The Swedish methadone maintenance program. Soc Med Aspect Drug Abuse. 2012;7(3):205-13. doi: 10.1007/978-94011-6320-0_19.

4. Schuckit MA. Treatment of opioid-use disorders. $N$ Engl J Med. 2016;375(4):357-68. doi:10.1056/NEJMra1604339. [PubMed: 27464203].

5. Amato L, Davoli M, Perucci CA, Ferri M, Faggiano F, Mattick RP. An overview of systematic reviews of the effectiveness of opiate maintenance therapies: Available evidence to inform clinical practice and research. J Subst Abuse Treat. 2005;28(4):321-9. doi: 10.1016/j.jsat.2005.02.007. [PubMed: 15925266].

6. Volpicelli JR, Alterman AI, Hayashida M, O'Brien CP. Naltrexone in the treatment of alcohol dependence. Arch Gen Psychiatry. 1992;49(11):876-80. doi: 10.1001/archpsyc.1992.01820110040006. [PubMed: 1345133].

7. Fareed A, Vayalapalli S, Stout S, Casarella J, Drexler K, Bailey SP. Effect of methadone maintenance treatment on heroin craving, a literature review.JAddictDis. 2011;30(1):27-38. doi:10.1080/10550887.2010.531672. [PubMed: 21218308].

8. Darker CD, Ho J, Kelly G, Whiston L, Barry J. Demographic and clinical factors predicting retention in methadone maintenance: Results from an Irish cohort. Ir J Med Sci. 2016;185(2):433-41. doi: 10.1007/s11845-015-1314-5. [PubMed: 26026953].

9. Fudalej S, Ilgen M, Kolodziejczyk I, Podgorska A, Serafin P, Barry K, et al. Somatic comorbidity and other factors related to suicide attempt among Polish methadone maintenance patients. J Addict Med. 2015;9(6):433-9. doi: 10.1097/ADM.0000000000000153. [PubMed: 26335004]. [PubMed Central: PMC4630090].

10. Beck AT, Steer RA, Ranieri WF. Scale for Suicide Ideation: Psychometric properties of a self-report version. J Clin Psychol. 1988;44(4):499-505. doi: 10.1002/1097-4679(198807)44:4<499::aidjclp2270440404>3.0.co;2-6. [PubMed: 3170753].

11. Kessler RC, Borges G, Walters EE. Prevalence of and risk factors for lifetime suicide attempts in the National Comorbidity Survey. Arch Gen Psychiatry. 1999;56(7):617-26. doi: 10.1001/archpsyc.56.7.617. [PubMed: 10401507].
12. Simon RI. Preventing patient suicide: Clinical assessment and management. Arlington, VA: American Psychiatric Pub; 2011.

13. Borges G, Walters EE, Kessler RC. Associations of substance use, abuse, and dependence with subsequent suicidal behavior. Am J Epidemiol. 2000;151(8):781-9. doi: 10.1093/oxfordjournals.aje.a010278. [PubMed 10965975].

14. Cottler LB, Campbell W, Krishna VA, Cunningham-Williams $\mathrm{RM}$, Abdallah $\mathrm{AB}$. Predictors of high rates of suicidal ideation among drug users. J Nerv Ment Dis. 2005;193(7):431-7. doi: 10.1097/01.nmd.0000168245.56563.90. [PubMed: 15985836]. [PubMed Central: PMC1350972].

15. Yuodelis-Flores C, Ries RK. Addiction and suicide: A review. Am JAddict 2015;24(2):98-104. doi: 10.1111/ajad.12185. [PubMed: 25644860].

16. Wormser GP, Park K, Madison C, Rozenberg J, McKenna D, Scavarda C, et al. Evaluation of prospectively followed adult patients with erythema migrans using the Beck depression inventory second edition. Am J Med. 2019;132(4):519-24. doi: 10.1016/j.amjmed.2018.11.039. [PubMed: 30562495]

17. Ghassemzadeh H, Mojtabai R, Karamghadiri N, Ebrahimkhani N. Psychometric properties of a Persian-language version of the Beck Depression Inventory-Second edition: BDI-II-PERSIAN. Depress Anxiety. 2005;21(4):185-92. doi:10.1002/da.20070. [PubMed:16075452].

18. Starosta AJ, Brenner LA. Beck Anxiety Inventory. In: Kreutzer J, DeLuca J, Caplan B, editors. Encyclopedia of clinical neuropsychology. Springer, Cham; 2017. doi: 10.1007/978-3-319-56782-2_1972-2.

19. Kaviani H, Mousavi AS. [Psychometric properties of the Persian version of Beck anxiety inventory (BAI)]. Tehran Univ Med J. 2008;66(2):136-40. Persian.

20. Rawlings D, Claridge G, Freeman JL. Principal components analysis of the Schizotypal personality scale (STA) and the borderline personality scale (STB). Pers Indiv Differ. 2001;31(3):409-19. doi: 10.1016/s01918869(00)00146-x.

21. Mashhadi A, Soltani E, Akbari E, Farmani A. The relationship between childhood attention deficit/hyperactivity disorder and adulthood borderline personality disorder. Zahedan J Res Med Sci. 2013;15(2):6873.

22. Vaux A, Phillips J, Holly L, Thomson B, Williams D, Stewart D. The social support appraisals (SS-A) scale: Studies of reliability and validity. Am J Community Psychol.1986;14(2):195-218. doi:10.1007/bfo0911821.

23. Shahry P, Kalhori SR, Esfandiyari A, Zamani-Alavijeh F. A comparative study of perceived social support and self-efficacy among women with wanted and unwanted pregnancy. Int J Community Based Nurs Midwifery. 2016;4(2):176-85. [PubMed: 27218115]. [PubMed Central: PMC4876786].

24. Hashim I. Cultural and gender differences in perceptions of stressors and coping skills. Sch Psychol Int. 2016;24(2):182-203. doi: 10.1177/0143034303024002004.

25. Yousefi F. [The relationship between emotional intelligence and communication skills in university students]. Dev Psychol (J Iran Psychol). 2006;3(9):5-13. Persian.

26. Carr ER, Woods AM, Vahabzadeh A, Sutton C, Wittenauer J, Kaslow NJ. PTSD, depressive symptoms, and suicidal ideation in African American women: A mediated model. J Clin Psychol Med Settings. 2013;20(1):37-45. doi: 10.1007/s10880-012-9316-1. [PubMed: 22802144] [PubMed Central: PMC3846783].

27. Steer RA, Kumar G, Beck AT. Self-reported suicidal ideation in adolescent psychiatric inpatients. J Consult Clin Psychol. 1993;61(6):1096-9. doi: 10.1037||0022-006x.61.6.1096. [PubMed: 8113489].

28. Esfahani M, Hashemi Y, Alavi K. Psychometric assessment of beck scale for suicidal ideation (BSSI) in general population in Tehran. Med J Islam Repub Iran. 2015;29:268. [PubMed: 26793659]. [PubMed Central: PMC4715388].

29. Fadardi S, Fodardi S, Baphrafan S. [Effectiveness of attention control training on reducing the attentiveness of attention to materials and improving the therapeutic indicators of addicts treated with detoxification]. Clin Psychol Res Counsel. 2011;11. Persian. 
30. Segrin C. Indirect effects of social skills on health through stress and loneliness. Health Commun. 2019;34(1):118-24. doi: 10.1080/10410236.2017.1384434. [PubMed: 29053380].

31. Xu YM, Zhong BL, Chen WC, Zhu JH, Lu J. Suicidal ideation among Chinese methadone-maintained patients: Prevalence and correlates. Oncotarget. 2017;8(49):86181-7. doi: 10.18632/oncotarget.21032. [PubMed 29156786]. [PubMed Central: PMC5689676].

32. Richardson L, Wood E, Montaner J, Kerr T. Addiction treatmentrelated employment barriers: The impact of methadone maintenance. J Subst Abuse Treat. 2012;43(3):276-84. doi 10.1016/j.jsat.2011.12.008. [PubMed: 22301085]. [PubMed Central: PMC3345299].

33. Hiswals AS, Ghilagaber G, Wijk K, Oberg P, Soares J, Macassa G. Employment status and suicidal ideation during economic recession. Health Sci J. 2015;9(1).

34. Blakely TA, Collings SC, Atkinson J. Unemployment and suicide. Evidence for a causal association? J Epidemiol Community Health. 2003;57(8):594-600. doi: 10.1136/jech.57.8.594. [PubMed: 12883065]. [PubMed Central: PMC1732539].

35. Zeng R, Cohen LJ, Tanis T, Qizilbash A, Lopatyuk Y, Yaseen ZS, et al. Assessing the contribution of borderline personality disorder and features to suicide risk in psychiatric inpatients with bipolar disorder, major depression and schizoaffective disorder. Psychiatry Res. 2015;226(1):361-7. doi: 10.1016/j.psychres.2015.01.020. [PubMed: 25661531].

36. Lutterman T, Hollen V, Shaw R. Substance abuse and mental health services administration (SAMHSA). Choice Rev Online. 2011;49(3):1507. doi: 10.5860/choice.49-1507.
37. Soloff PH, Pruitt P, Sharma M, Radwan J, White R, Diwadkar VA. Structural brain abnormalities and suicidal behavior in borderline personality disorder. J Psychiatr Res. 2012;46(4):516-25. doi: 10.1016/j.jpsychires.2012.01.003. [PubMed: 22336640]. [PubMed Central: PMC3307855].

38. Pirnia B, Asadi R. Examining the relationship between borderline personality traits and the level of craving for methamphetamine use among men and women. Int I Indian Psychol. 2015;3(1)

39. Wang Y, Wang H, Li W, Zhu J, Gold MS, Zhang D, et al. Reduced responses to heroin-cue-induced craving in the dorsal striatum: Effects of long-term methadone maintenance treatment. Neurosci Lett. 2014;581:120-4. doi: 10.1016/j.neulet.2014.08.026. [PubMed: 25157798].

40. Brent DA, Melhem N. Familial transmission of suicidal behavior. Psychiatr Clin North Am. 2008;31(2):157-77. doi: 10.1016/j.psc.2008.02.001. [PubMed: 18439442]. [PubMed Central: PMC2440417].

41. Zhang X, Xu H, Gu J, Lau JT, Hao C, Zhao Y, et al. Depression, suicidal ideation, and related factors of methadone maintenance treatment users in Guangzhou, China. AIDS Care. 2016;28(7):851-6. doi: 10.1080/09540121.2015.1124981. [PubMed: 26839180].[PubMed Central: PMC5266602].

42. Klonsky ED, Saffer BY, Bryan CJ. Ideation-to-action theories of suicide: A conceptual and empirical update. Curr Opin Psychol. 2018;22:38-43. doi: 10.1016/j.copsyc.2017.07.020. [PubMed:30122276].

43. May AM, Victor SE. From ideation to action: recent advances in understanding suicide capability. Curr Opin Psychol. 2018;22:1-6. doi: 10.1016/j.copsyc.2017.07.007. [PubMed: 30122270]. [PubMed Central: PMC6103644] 